\title{
LA GOBERNANZA DE REDES SOCIOEDUCATIVAS: CLAVES PARA UNA GESTIÓN EXITOSA
}

\author{
The governance of social-educative networks: keys \\ to successful management
}

\section{La gouvernance de réseaux socio-éducatifs: les clés de la gestion reussie}

Jorge Díaz Gibson, Mireia Civís Zaragoza y Jordi Longás Mayayo Universidad Ramon Llull. Facultad de Psicología y Ciencias de la Educación y del Deporte, Blanquerna. C/ Císter, 34.08022 Barcelona, España. Correo-e: jorgedg@ blanquerna.url.edu; mireiacz@blanquerna.url.edu; jordilm@blanquerna.url.edu

Fecha de recepción: marzo de 2013

Fecha de aceptación: junio de 2013

Biblid [(1130-3743) 25, 2-2013, 213-230]

\section{RESUMEN}

El presente trabajo plantea un modelo de gestión de redes socioeducativas (RS) basado en la colaboración interinstitucional y la transversalidad de la acción socioeducativa a nivel comunitario. Investigadores reconocidos afirman que la gestión de redes organizacionales difiere significativamente de los modelos aplicados a una institución socioeducativa tradicional e independiente. Sin embargo, la literatura muestra un importante vacío respecto a cómo gestionar de forma global la complejidad de estas estructuras organizativas para maximizar sus posibilidades de éxito. Los resultados del presente trabajo muestran un marco integrador de los factores críticos en la gestión de Rs que las conducen hacia su efectividad, a través del desarrollo de una cultura profesional colaborativa y el incremento del capital social en la comunidad. De esta manera, el modelo presentado concreta unas pautas para la orientación y evaluación de la función de gobernanza en las Rs. 
Palabras clave: redes socioeducativas, gobernanza, colaboración, gestión transversal, cultura colaborativa, capital social.

\section{SUMMARY}

This paper presents a social-educative networks managerial model based on interorganizational collaboration across the community. Researchers underline significant differences between network management and managerial issues from an independent and traditional organization. But literature shows an important lack around the specific managerial variables needed to lead these complex initiatives, in order to maximize goal achievement in a community level. Results from this work present a global frame by integrating crucial strategies in the management of socialeducative networks, as process steps that drive the network performance to its effectiveness through the development of a collaborative culture between professionals in the community, and the enhancement of community social capital. Therefore, the model provides some specific guidelines for network managers, so as a frame for the assessment of managerial performance.

Key words: social-educative networks, governance, collaboration, transversal management, collaborative culture, social capital.

\section{SOMMAIRE}

Ce document propose un modèle de gestion des réseaux socio-éducatifs (RS) basée sur la collaboration interinstitutionnelle et sur la transversalité de l'action socio-éducative au niveau communautaire. Des reconnus chercheurs affirment que la gestion des réseaux organisationnels diffère sensiblement des modèles appliqués sur des institutions socio-éducatives traditionnelles et indépendantes. A la fois, la littérature montre qu'il existe un manque significatif d'études sur comme gérer globalement la complexité de ces structures organisationnelles afin de maximiser leurs chances de réussite. Les résultats de ce travail montrent un cadre qui intègre les facteurs critiques dans la gestion des Rs et qui augmente son efficacité à travers le développement d'une collaborative culture professionnelle et d'augmenter le capital social dans la communauté. Ainsi, le modèle présenté spécifie des lignes directrices pour l'orientation et l'évaluation du rôle de la gouvernance dans des RS.

Mots-clés: réseaux socio-éducatives, gouvernance, la collaboration, gestion transversale, culture de collaboration, capital social. 


\section{INTRODUCCIÓN}

La naturaleza compleja e interdependiente de los retos socioeducativos actuales requiere un enfoque sistémico y estratégico de las políticas públicas. Incidir en la mejora del éxito escolar, reducir la pobreza infantil, promover el acceso de los jóvenes al trabajo o mejorar los hábitos de vida saludable, ineludibles prioridades en la agenda política de cualquier país occidental, son desafíos interconectados que no pueden abordarse de forma aislada o parcializada. Los problemas sociales responden a múltiples variables que forman un entramado complejo, atravesados por una dimensión comunitaria que rompe las barreras organizacionales de las propias instituciones socioeducativas, hasta el punto de que la colaboración interinstitucional se convierte en un requisito imprescindible para abordarlas con éxito (Milward y Provan, 2006).

Como consecuencia, se explica que en las últimas décadas hayan emergido paralelamente diversas experiencias de trabajo o acción socioeducativa en red para atender las necesidades en el ámbito local y/o comunitario. La evaluación de dichas experiencias, como las Zones d'action Prioritaire (Francia, 1982), las Education Action Zones (Reino Unido, 1998), las Extended Schools (Reino Unido, 2004), The Harlem Children's Zone (Estados Unidos, 1990) o Interxarxes y Redes Educativas Locales (España, 2000), han ido nutriendo y capacitando estas redes como modelo de organización-acción en el ámbito socioeducativo, expandiendo su esencia y mejorando cualitativamente sus prácticas hasta la actualidad.

Definimos las redes socioeducativas (RS) como formas estables de colaboración entre organizaciones públicas y privadas para afrontar objetivos socioeducativos comunes, así como para formular e implementar programas (Agranoff y McGuire, 2001), desarrollando una estructura propia de coordinación-acción a nivel comunitario. Las RS se construyen mediante la implicación de las instituciones y entidades locales, basan su acción en la colaboración a nivel comunitario y buscan dar respuestas sistémicas e integradas a los retos socioeducativos planteados. Su estudio ha sido abordado paralelamente por investigadores europeos (governance networks -redes de gobernanza-) y estadounidenses (organizational networks -redes organizacionales-) desde el campo científico de la gestión pública, interesados en analizar las formas estables de colaboración interorganizacional como estrategias de acción a nivel comunitario

La literatura muestra resultados significativos logrados por las RS a partir de diferentes estudios de caso aplicados a prácticas relevantes, como son la mejora del éxito escolar en diversas comunidades de Inglaterra y Estados Unidos (Carpenter et al., 2010; Renée y McAllister, 2011), la mejora de la capacidad de innovación del sector público en Dinamarca (Sorensen y Torfing, 2010) o la optimización de recursos socioeducativos a nivel comunitario, así como el incremento de la eficiencia organizativa en España (Ubieto, 2009; Díaz-Gibson, Civís, Longás y López, 2010 y 2012). En estos estudios cabe subrayar la unanimidad en destacar la "gestión activa" de las Rs como factor clave de su sostenibilidad y éxito. Sólo 
donde se invierten esfuerzos y energía explícitamente dirigida a coordinar la acción y estimular la colaboración entre las distintas instituciones socioeducativas se obtienen resultados relevantes.

Sin embargo, aunque la gestión de redes es un campo emergente dentro de la gestión pública y se reconoce su carácter específico o distinto de los modelos clásicos de gestión (Agranoff y McGuire, 1999; Bourgault y Lapierre, 2000; Agranoff, 2003; Sorensen y Torfing, 2009), la literatura profundiza poco en la naturaleza del trabajo necesario para alcanzar los objetivos de la red en su globalidad (Provan, Fish y Sydow, 2007; Renée y McAlister, 2011). Para Kickert, Klijn y Koppenjam (1997), la gestión de las RS debe orientarse preferentemente a mejorar la habilidad de la red para el cumplimiento de sus objetivos y, a su vez, facilitar las interacciones entre sus miembros. Milward y Provan (2006) confirman que algunas estrategias de gestión, como la gestión del diseño, del conflicto o del compromiso, inciden en la eficiencia de las RS. A pesar de éstos, faltan trabajos que definan la relación entre estrategias de gestión y modelos de acción en red (Carpenter et al., 2010) y que a su vez nos ayuden substancialmente a comprender y acotar la gestión de las RS.

Este artículo propone un modelo de gestión de las Rs que maximice el logro de objetivos a través de la promoción de una cultura profesional colaborativa y la construcción de capital social a nivel comunitario. Para desarrollar el modelo, se contrasta el marco analítico que vincula las Rs con la promoción de capital social (Díaz-Gibson y Civís, 2011) con la literatura internacional sobre gestión de redes, concluyendo con una propuesta de modelo que acota y organiza las estrategias de gestión. Con ello, los gestores de las RS disponen de pautas que orientan su función de liderazgo en este tipo de organización desde criterios de efectividad -mejora del impacto de sus prácticas y logro de los objetivos en la comunidad- y se concreta un hipotético marco de evaluación de su propia actividad.

\section{LA GOBERNANZA DE REDES SOCIOEDUCATIVAS Y EL CAPITAL SOCIAL}

La revisión de la literatura sobre RS nos muestra tres concepciones que nutren su esencia y caracterización: a) las redes interorganizacionales, que incorporan el énfasis en la independencia general de las instituciones participantes (Milward y Provan, 2006); b) las redes de gobernanza, que destacan la diversidad de los miembros y su discusión como fuente de riqueza (Klijn, Edelenbos y Steijn, 2010); y c) las organizaciones comunitarias o community partnerships, que enfatizan en las alianzas desde un enfoque comunitario como marco de acción (Shirley, 2009). Entendemos que estas tres perspectivas nos aportan una conceptualización complementaria e integradora (Tabla 1). En consecuencia, definimos las RS como redes organizacionales de carácter formal, integradas por distintos actores (instituciones y entidades públicas y privadas), orientadas hacia el logro de metas socioeducativas comunes, que basan su acción en la colaboración a través de una programación estratégica conjunta y que son promotoras de capital social en la comunidad. 


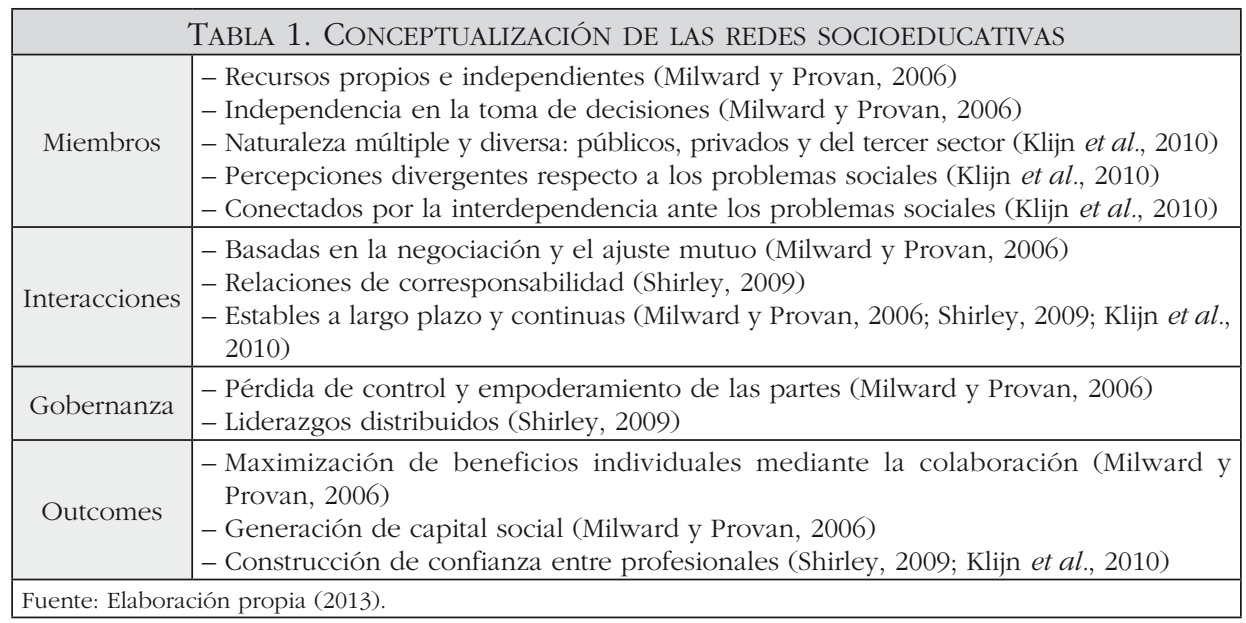

Un aspecto de sumo interés en el estudio de las RS es su relación con la gobernanza. En primer lugar porque existe un reconocimiento entre los expertos de la validez de la acción socioeducativa en red como estrategia de gobernanza pública para afrontar los retos de la comunidad. Y, en segundo lugar, porque la propia gestión de las Rs es un ejercicio de gobernanza -O metagovernance (Sorensen y Torfing, 2009)-, en la medida que son generadoras de sinergias y colaboración entre los diferentes actores socioeducativos que inciden en una comunidad. Este enfoque, además de responder a una nueva mirada de la acción, supone también una nueva concepción organizativa de las políticas. De acuerdo con algunos autores, aseveramos que la gobernanza socioeducativa mediante el desarrollo de Rs favorece la democratización de las decisiones en la comunidad, así como el afianzamiento de la colaboración entre los diferentes actores afectados, algo que se ha convertido en requisito básico para alcanzar el éxito de las políticas trazadas (Gray, 2000; Sorensen y Torfing, 2010).

Pero desde la perspectiva de la gobernanza, la cuestión principal no es conocer cómo y hasta qué punto la construcción conjunta de conocimiento y el consenso en la toma de decisiones ayuda a mejorar la acción socioeducativa, sino cuál es la efectividad -eficiencia y eficacia- de estas formas de organización y cuáles son sus claves. Y, al respecto, entendemos que actualmente existen ciertas limitaciones a la hora de evaluar la efectividad de las Rs, debido a su complejidad inherente, a la falta de referentes empíricos en la literatura internacional y a las limitaciones en la atribución directa de un resultado comunitario a una acción determinada. Si revisamos los informes finales de las evaluaciones de los Planes Educativos de Entorno en Cataluña (2005-2009) o de las Extended Schools en Reino Unido (20042011), ambos concluyen que los resultados finales no son satisfactorios en relación al objetivo de mejorar el rendimiento escolar. A su vez, ambos informes apuntan que si bien parecen lograrse avances en aspectos comunitarios -nuevas dinámicas 
colaborativas, mejoras en la participación, cohesión social, etc.-, éstos no han sido evaluados ni considerados como mejoras explícitas u outcomes.

Pensamos que tanto en estas como en otras experiencias de Rs, la variable tiempo puede ser crítica y que debería jugar a favor de aquellos procesos que se consolidan y desarrollan debidamente. De hecho, en nuestras investigaciones anteriores planteamos para la evaluación de las Rs, más allá del logro de objetivos, virar hacia el concepto de éxito entendido como sostenibilidad de las experiencias y alta valoración por parte de los profesionales que integran las Rs (Díaz-Gibson, Civís, Longás y López, 2010). La finalidad de esta propuesta era medir el impacto global que aportaban estas experiencias en la comunidad. La literatura muestra cómo la naturaleza organizacional y colaborativa de estas redes contribuye por sí misma a la generación de valor público (Bryson, Crosby y Stone, 2006; Sorensen y Torfing, 2007), resultando mejoras en el capital social y en la confianza entre sus miembros (Gray, 2000; Agranoff y McGuire, 2003), e incrementándose el proceso de aprendizaje entre profesionales (Hajer y Wagenaar, 2003). Las investigaciones al respecto concluyen que estas mejoras aumentan las capacidades de la red y su eficiencia en el logro de objetivos, aportan soluciones más precisas e integrales (Agranoff y Mcguire, 2001; Edelenbos y Klijn, 2006; Sorensen y Torfing, 2007) y desarrollan progresivamente la capacidad para afrontar problemas más complejos (Kamensky, Burlin y Abramson, 2004; Rainey, 2009; Scearce, 2010).

En consecuencia, las evaluaciones que pretendan determinar la eficiencia y eficacia de las Rs no pueden obviar el impacto comunitario de unas estructuras que integran la inclusión, la diversidad, la colaboración y la corresponsabilidad como valores activos. En nuestros trabajos anteriores ya consideramos la madurez de las RS en relación a seis principios clave -proximidad, colaboración, horizontalidad, corresponsabilidad, transversalidad y proyección- y su incidencia sobre variables concretas de capital social como son la confianza, afiliación/implicación, voluntariado, organización comunitaria, compromiso con la educación, inclusión, diversidad y gestión/generación de conocimiento (Díaz-Gibson y Civís, 2011). De esta manera, somos partidarios de incorporarlos como outcomes comunitarios de las Rs que empoderan tanto a los profesionales como a los miembros de la comunidad, maximizando las posibilidades de lograr los objetivos socioeducativos planteados.

\section{LA GESTIÓN ACTIVA, MOTOR DEL ÉXITO DE LAS REDES SOCIOEDUCATIVAS}

Las conclusiones de algunos de los trabajos empíricos sobre Rs llevados a cabo en Cataluña aportan datos relevantes en torno a su gestión. Un estudio realizado por los autores en 2008 por encargo del Instituto Municipal de Educación de Barcelona (IMEB) nos permitió conocer 30 experiencias de RS distintas ubicadas en 4 distritos de la ciudad. Mediante un mapeo y análisis cualitativo de estas prácticas llegamos a la conclusión de que las redes que carecían de un equipo de gestión carecían también de un motor que alimentara sus interacciones, su estructura era más frágil 
y su acción resultaba menos influyente, corriendo el constante riesgo de ver cómo disminuía su actividad hasta la desmembración. Más allá de la problemática de la supervivencia de la red (López, 2008), detectamos que las Rs más sostenibles y estables eran aquellas que destinaban parte de su presupuesto y energía a la gestión de las relaciones entre los actores que constituyen la red, algo que permitía maximizar los beneficios tanto de las instituciones miembros como de la propia RS.

Dado que la gestión es un factor crítico en el éxito de las RS, cabe interrogarse sobre sus características y la posible existencia de normas o principios que la orienten. Siguiendo a Agranoff y Mcguire (2003), la gestión de las redes organizacionales responde a un modelo de gestión no jerárquica, donde la información compartida y la experticia de los miembros permiten substituir la gestión y estructura autoritaria por procesos de autogestión y consenso. La peculiar constitución de las Rs -que sus miembros sean profesionales, pero también instituciones u organizaciones independientes- requiere una gestión que trascienda las fronteras de estas organizaciones para perseguir metas comunes. Dicho proceso, de algún modo aparentemente contrario a la autonomía jurídica y organizativa de cada miembro, incluye un doble objetivo que se retroalimenta y sustenta el éxito: a) la gestión de la estructura ha de aumentar la colaboración y debe incluir tareas como la reflexión sobre cómo o cuál es la mejor estructura para hacer frente a los retos; y b) la gestión de la acción global ha de perseguir la implementación del programa acordado y la efectividad en el logro de los objetivos.

Las RS son formas organizacionales de alta complejidad y están formadas por miembros de naturaleza dispar, con objetivos diversificados, culturas de trabajo que incluso en ocasiones pueden ser opuestas, ubicación en sectores o ámbitos dispares, personas con diferentes niveles o rangos en sus organizaciones, e incluso niveles distintos de la administración pública. Esta diversidad, inherente a las RS, supone una gran riqueza pero también un gran reto para su gestión. Por ello, una de las funciones de la gestión será alimentar todo aquello que conecta a todos sus miembros, como los intereses comunes, el público destinatario de la acción o la perspectiva comunitaria de los retos socioeducativos. La literatura recoge algunas de las tensiones a las que debe hacer frente la gestión de redes, fruto de esta circunstancia, para aumentar su efectividad: legitimidad interna versus externa, eficiencia versus inclusión, flexibilidad versus estabilidad (Provan y Kenis, 2008), y unidad versus diversidad (Saz y Ospina, 2010).

Además también debe considerarse que las Rs generan las condiciones necesarias para aumentar la innovación interorganizacional (Mandell y Steelman, 2003; López, 2008), especialmente cuando incluyen actores políticos y sociales con un alto grado de diversidad (Dente, Bobbio y Spada, 2005; Sorensen y Torfing, 2007). Esta capacidad, esencial para afrontar la incertidumbre de los nuevos desafíos socioeducativos y que está vinculada directamente con la colaboración (Sorensen y Torfing, 2010), influye directamente en la efectividad en el logro de los objetivos y en la generación de capital social, dotando a la comunidad de una mayor capacidad para organizarse, proveer servicios y desarrollar proyectos socioeducativos. 
Nuestros hallazgos, así como la importancia que otorga la literatura al papel de la gestión en el desarrollo de la acción de las RS y el cumplimiento de objetivos, nos llevan a definir un marco más acotado para su exitosa gestión. Concretamente, entendemos que las Rs pueden maximizar sus logros en la medida que las estrategias de gestión se orientan hacia el máximo desarrollo de los principios que hemos mencionado como constitutivos. Es decir, si la proximidad (respuesta a las necesidades reales e integrales de las personas del territorio), colaboración (construcción y enriquecimiento de conexiones entre los actores), horizontalidad (liderazgo compartido, igualdad entre actores, participación y consenso en la toma de decisiones), corresponsabilidad (compromiso mutuo y acción compartida), transversalidad (integración de la diversidad en la planificación e implementación del programa) y proyección (planificación estratégica e innovación) son principios concebidos como metas que guíen la acción global de las Rs, ésta caminará hacia los logros organizacionales y sociales necesarios para el logro de objetivos finales.

\section{GESTIÓN HORIZONTAL, VERTICAL Y CAMBIO EN LA CULTURA PROFESIONAL}

Hemos visto que el éxito de las Rs depende significativamente del acierto en su gestión, es decir, de cómo la función gestora -vital en cualquier tipo de organización- es capaz de activar y articular los procesos colaborativos entre sus miembros. Por consiguiente, la pregunta que corresponde abordar es la referida a las necesidades o requisitos que debe satisfacer la gestión de las Rs. A tal fin, consideramos que existen tres dimensiones fundamentales para el gobierno de redes organizacionales (Díaz-Gibson et al., 2013): a) la estructura, entendida como el modo como se organizan y gestionan las interacciones, b) la acción, siendo el modo como se gestiona la implementación global del proyecto y c) la cultura, entendida como los valores, actitudes y comportamientos profesionales de los miembros de la red, y que necesariamente debe avanzar hacia una cultura colaborativa.

Las necesidades de gestión generadas por la singular estructura y tipo de interacciones de las RS dan lugar al vector que llamamos gestión horizontal. Esta gestión es la más descrita en la literatura y se centra en los procesos necesarios para facilitar las interacciones colaborativas, y así alcanzar los objetivos de la red. Algunos autores definen esta acción general como Facilitar-Facilitating- (Saz y Ospina, 2009) o Sintetizar -Synthethising- (Agranoff y McGuire, 2001). Para Milward y Provan (2006) consiste en gestionar de forma propositiva: el conflicto entre sus miembros, la responsabilidad y el compromiso con las metas comunes, la legitimidad desde la participación colectiva y el diseño de la estructura -como las acciones destinadas a asignar una estructura de gobierno óptima para el logro de objetivos-. La gestión horizontal que necesita una RS exitosa está representada por el concepto de metagobernanza (Sorensen y Torfing, 2009), que ilustra la gobernanza de la red como estrategia de gobernanza local, e incluye los intentos deliberados para capacitar y dirigir la autorregulación de los procesos de interacción 
colaborativa, sin volver a los estilos tradicionales de gobierno en términos de burocracia, reglamentación y mandato imperativo. De esta manera, el consenso se convierte en el proceso para la toma de decisiones a través de los espacios de la red, con el triple objetivo de sacar partido de la diversidad de sus miembros, lograr una alta representatividad en las decisiones y alcanzar la máxima transversalidad en la acción.

El segundo vector, que denominamos gestión vertical, responde a las necesidades de tipo operativo de las RS, es decir, la forma de abordar la acción global de la red en su propósito de lograr los objetivos finales que se han fijado. Algunos estudios señalan que existe la necesidad de gestionar las acciones colectivas, utilizando diferentes conceptos como la gestión interdepartamental, gestión de las alianzas o gestión de las acciones conjuntas (Bourgault y Lapierre, 2000). La literatura concreta algunas de las especificaciones para organizar la acción de la red, como son el trabajar en diferentes niveles de implementación (Mediratta, Shah y McAlister, 2009), la búsqueda de alianzas a través de la comunidad (Gold, Simon y Brown 2002) y la construcción de equipos colaborativos e interdisciplinares (DíazGibson et al., 2013). Finalmente, para incrementar la calidad de la acción también son estrategias clave la formación de los miembros de las RS, las evaluaciones formales -tanto de la acción global como de la acción gestora- e implementar mecanismos de mejora continua (Lipnack y Stamps, 1994).

Respecto a la dimensión cultural de las RS y sus necesidades de gestión son pocas las referencias encontradas en la literatura. No obstante, desde nuestra perspectiva su gestión es clave y reúne aquellas tareas dirigidas a transformar el modo de entender y desarrollar la profesionalidad socioeducativa. Diversos investigadores coinciden en afirmar que para sacar rendimiento de la diversidad de los miembros de una RS y establecer un proyecto común basado en el compromiso voluntario, así como un diseño de gobierno democrático, es necesaria una cultura de trabajo colaborativa (Hjern, 1992; Kickert et al., 1997; Milward y Provan, 2006; Sorensen y Torfing, 2007). A su vez, algunos autores identifican determinados aspectos de la cultura profesional y de la política interna de las instituciones como barreras para el desarrollo de RS. Para Eggers y Singh (2009) el silo thinking -la reflexión cilíndrica o departamentalista- se convierte en un obstáculo importante en la resolución de los problemas que afrontan las redes colaborativas. Se constata que la acción socioeducativa colectiva o comunitaria supone un reto difícil para los profesionales, generalmente acostumbrados al trabajo individual y departamentalizado, que a lo sumo requiere de coordinaciones esporádicas entre servicios. Esta tradición profesional y organizacional, a la que Ubieto (2009) denomina modelo de la reingeniería, limita la capacidad de respuesta ante las demandas hoy en día emergentes, que requieren del trabajo colaborativo entre diversas instituciones y profesionales de la acción social y educativa. Esta realidad nos lleva a plantear la necesidad de trabajar por una "nueva" cultura profesional entre los actores socioeducativos (Ubieto, 2009; Díaz-Gibson et al., 2010). 
A favor de este cambio cabe destacar el interés mostrado por los profesionales que tienen la oportunidad de trabajar de forma colaborativa o en red, según muestran las conclusiones de múltiples estudios de caso realizados en diferentes países. En ellos se constata que los actores valoran especialmente las ventajas profesionales que aporta la colaboración, tanto por el enriquecimiento mutuo, como por el enriquecimiento de la respuesta global (Ubieto, 2009; Díaz-Gibson et al., 2010; Renée y McAlister, 2011). De este modo, los propios profesionales implicados en la acción socioeducativa comunitaria sitúan a las RS como motor del cambio hacia una cultura profesional más crítica, interactiva, dinámica y reflexiva (Shön, 1998). Así lo confirman Renée y Mcalister (2011) cuando explican como gran parte de las RS en Estados Unidos tienen como objetivo principal la transformación de la cultura de las escuelas, de manera que padres, maestros, directores y el resto de la comunidad trabajen conjuntamente por el beneficio de los niños y adolescentes.

Estas tres dimensiones que debe satisfacer la gestión de las RS -horizontal, vertical y cultural- están interconectadas entre sí y, de algún modo, se retroalimentan. Es decir, tanto el fomento de la colaboración como la implementación del proyecto deben generar un avance o cambio significativo hacia una cultura colaborativa profesional; a la vez que ésta facilitará el desarrollo de dicha gestión. De esta manera situamos la dimensión cultural de la red en el horizonte de la gestión, entendiendo que una cultura profesional más colaborativa permitirá a la función gestora armonizar las necesidades de horizontalidad y transversalidad con las necesidades de eficacia y eficiencia en el logro de objetivos.

\section{El MODELO DE GESTIÓN TRANSVERSAL DE REDES SOCIOEDUCATIVAS}

En la aproximación teórica a la gestión de las Rs hemos destacado la importancia de la función gestora como aspecto crítico para conseguir la máxima efectividad de estas complejas formas de gobernanza socioeducativa. Con el fin de facilitar un marco teórico que oriente la función gestora, a continuación proponemos el Modelo de Gestión Transversal de Rs (Gráfico 1), un modelo global que integra la triple dimensión de necesidades internas que debe satisfacer la gestión, así como los indicadores que concretan la generación de capital social en la comunidad. De esta manera, el modelo integra seis estrategias de gestión que representan los "nuevos" valores que fundamentan el necesario viraje en la cultura profesional, orientados al desarrollo comunitario y a la eficiencia de la acción colectiva. 


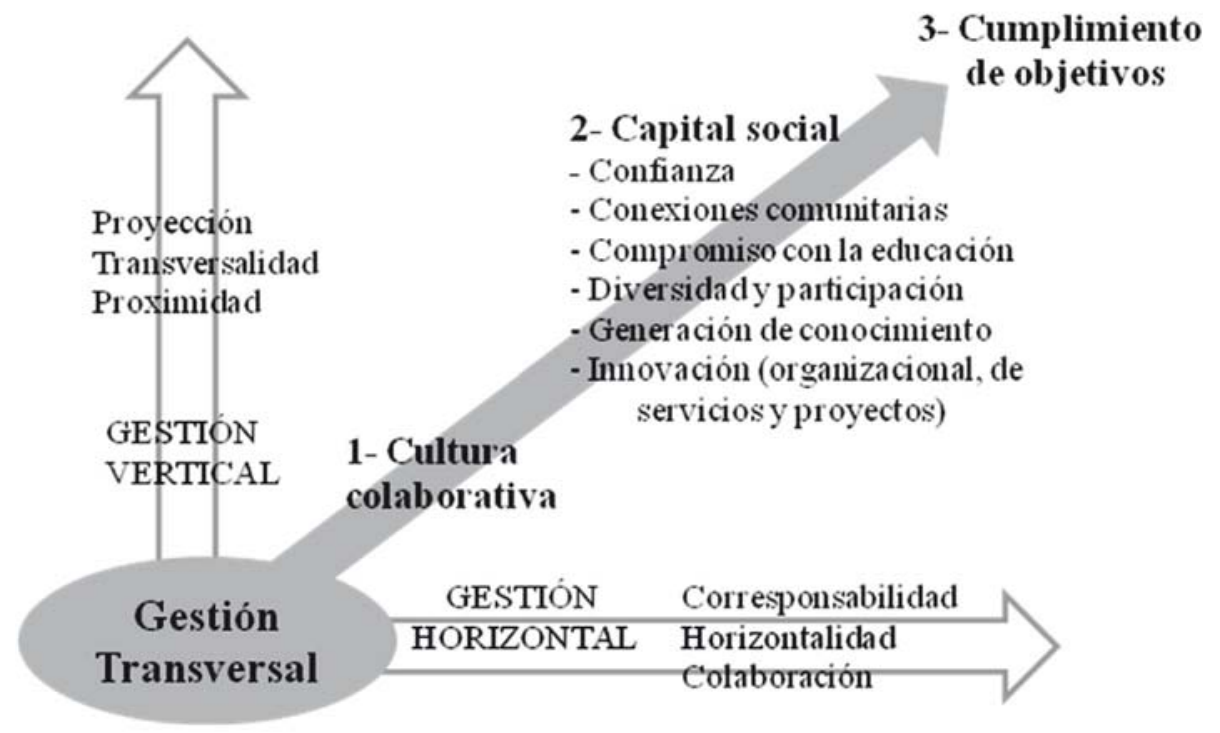

Fuente: Elaboración propia (2013).

Como ilustra el Gráfico 1, el modelo persigue una mayor efectividad en la consecución de sus objetivos, previo desarrollo de una cultura profesional colaborativa y del fortalecimiento del capital social en la comunidad. De esta manera, se apuntan las estrategias de gestión en sus dimensiones horizontal y vertical, donde un mayor cumplimiento de las mismas nos reportará un mayor logro en las metas tanto a nivel profesional -desarrollo de una cultura colaborativacomo comunitarias -incremento del capital social-.

La Gestión Horizontal, entendida como aquella focalizada en la dimensión estructural de la red, está orientada a generar y capacitar las conexiones entre sus miembros, desarrollando las condiciones específicas para la acción global. Ésta incluye las estrategias centradas en procesos interactivos dirigidos a la cohesión de unas estructuras complejas y diversas como son las Rs. Estas estrategias de gestión son la Corresponsabilidad, la Horizontalidad y la Colaboración, y a continuación pasaremos a desarrollar sus componentes esenciales así como su prevalencia a través de la literatura.

La estrategia de la Corresponsabilidad es subrayada por la literatura como una acción necesaria para alimentar la unión del círculo común entre todos los miembros de la red. La construcción de una visión y un propósito colectivo son la clave para el éxito de la red (Lipnack y Stamps, 1994; Shirley, 2009). De esta manera, 
la corresponsabilidad no es solo un valor axiológico que favorece la creación y la sostenibilidad de las RS, sino una estrategia que se tiene que alimentar durante el proceso. Algunos autores apuntan la importancia de nutrir aquello que une a los diversos miembros (Kamensky et al., 2004), estableciendo un seguimiento de la mutua obligación para mejorar los resultados socioeducativos en la comunidad. Skelcher y Torfing (2010) añaden que para alimentar la corresponsabilidad y mejorar la colaboración es necesario promover la propiedad conjunta de los outputs de la red, entendiendo que todo aquello surgido de la red es propiedad de todos. El trabajo colaborativo y el desarrollo de un proyecto común entre los diferentes miembros de la red -maestros, educadores sociales, directores de escuela, inspectores educativos, investigadores u otros partners- requieren de un soporte y unión que agrupe las propuestas individuales (Renée y McAlister, 2011). A su vez, esta estrategia está altamente ligada al desarrollo de capital social y concretamente a su variable de compromiso con la educación, en tanto que atrae a diversos sectores sociales no estrictamente educativos, a trabajar activamente para alcanzar metas socioeducativas en la comunidad.

La Horizontalidad pretende establecer unas relaciones democráticas e igualitarias y por tanto será necesaria la habilidad en la gestión para construir consensos (Kamensky et al., 2004). Para gestionar la diversidad de los miembros en la red y establecer un proyecto común basado en el compromiso voluntario con las finalidades, es necesario un diseño basado en una gobernanza democrática con una distribución de poder igualitaria (Hjern, 1992; Kickert et al., 1997; Sorensen y Torfing, 2000 y 2007; Agranoff, 2003; Milward y Provan, 2006). Sorensen y Torfing (2009) utilizan el concepto de metagovernance para identificar un elemento clave de la gestión de redes, como la dirección de procesos más o menos autorregulados de interacción colaborativa sin caer en estructuras de tomas de decisión tradicionales y jerárquicas. Por otro lado, la horizontalidad tiene como finalidad la promoción de un rol de liderazgo dinámico y distribuido entre sus miembros. Kamensky et al. (2004) señalan la existencia de múltiples líderes en la red como un indicador de su éxito. A su vez, Lipnack y Stamps (1994) apuntan el valor agregado y la resiliencia que aportan múltiples líderes a la red, entendiendo que cada miembro tiene algo único que compartir durante el proceso.

La estrategia dirigida a incrementar la Colaboración en los procesos de trabajo conjunto es una acción destacada a través de la literatura, tanto en el aumento del número de encuentros como en el de la calidad de los vínculos entre los miembros. Algunos investigadores comparten la idea de que para llevar a cabo los objetivos de la Red es necesaria una cultura colaborativa (Kickert et al., 1997; Milward y Provan, 2006; Sorensen y Torfing, 2007; Renée y Mcalister, 2011). Cigler (1999) definió la colaboración como la existencia de vínculos de una gran intensidad, e incluía acciones como el incremento de recursos compartidos, de actividades conjuntas y de objetivos comunes. Kamesnsky (2004) añadía aspectos importantes en la colaboración como la planificación conjunta, el esfuerzo colectivo y la propiedad común de todo aquello surgido del trabajo colaborativo. Esta estrategia 
es también un generador de confianza (Lipnack y Stamps, 1994) que facilita una visión conjunta y una acción global eficiente (Klijn, Edelenbos y Steijn, 2010). Finalmente, O'Leary (2009) describe cómo los gestores de redes deben tener la habilidad comunicativa para gestionar el conflicto de forma positiva, ya que éstos están muy presentes en la red y pueden obstruir el trabajo colectivo si no son guiados y entendidos de forma constructiva (Milward y Provan, 2006).

Tanto la estrategia de Colaboración como la de Horizontalidad están estrechamente conectadas al desarrollo de capital social a través de la generación de confianza. Por tanto, dichas estrategias concretan pautas para la construcción de confianza a nivel comunitario -entre profesionales, entre instituciones o servicios y entre los miembros de la comunidad que participan en la red-, facilitando así la eficiencia global de la red (Klijn, Edelenbos y Steijn, 2010).

Por otro lado, la Gestión Verticalagrupa las tareas dirigidas a articular la actuación colectiva y por tanto se refiere a la gestión de la dimensión de la acción en la red. Esta gestión se centra en el diseño e implementación de los proyectos a través de la gestión de todos los espacios de toma de decisiones en la red. Además, la gestión vertical de las Rs incluye acciones estratégicas como la contextualización comunitaria de las prácticas, la búsqueda de compromiso y participación comunitaria o la planificación a largo plazo. Dicha gestión nos conduce, a través de la colaboración, a la construcción de una cultura de innovación continua en el ámbito socioeducativo. Seguidamente, pasamos a describir las estrategias de Transversalidad, Proximidad y Proyección que permiten concretar la Gestión Vertical.

La Transversalidad tiene como objetivo la integración y articulación de la diversidad en la Rs para la planificación e implementación del proyecto común. Esta estrategia va dirigida a desarrollar una planificación que atraviese las fronteras de las organizaciones miembros (Lipnack y Stamps, 1994). La diversidad aparece como un activo importante para capacitar la acción en la comunidad (Krebs y Holley, 2004), multiplicando las opciones de respuesta a los problemas que aportan distintas miradas socioeducativas que provienen de diversas disciplinas, ámbitos y sectores. Las redes funcionan a diferentes niveles de acción (Lipnack y Stamps, 1994), de manera que una acción global y articulada requiere trabajar en diferentes niveles de implementación al mismo tiempo (Mediratta, Shah y McAlister, 2009). El diseño de estos espacios de toma de decisiones sobre la acción es conocida por algunos autores como estructurar-framing-(Agranoff y McGuire, 2001; Saz y Ospina, 2009), de modo que se diseñan los espacios de colaboración. Por consiguiente, la Transversalidad persigue el máximo aprovechamiento de recursos en la comunidad. Los últimos estudios sobre la gestión de la Transversalidad en RS nos indican la importancia de las necesidades socioeducativas de la comunidad y los objetivos comunes como determinantes del proyecto de red, además de la gestión de equipos colaborativos e interdisciplinares en tres espacios definidos: las Asambleas, de participación global y establecimiento de prioridades generales; las Comisiones, focalizadas en las líneas estratégicas; y los Equipos de trabajo, centrados en la implementación del proyecto (Díaz-Gibson et al., 2013). Finalmente, esta estrategia está conectada al desarrollo de 
capital social mediante la variable de diversidad y participación. Dicho argumento se basa en la idea de que las asociaciones con un alto grado de heterogeneidad entre sus miembros constituyen, en sí mismas, una plataforma para la vinculación entre clases socioeconómicas divergentes (Stolle y Rochon, 1998; Putnam, 2000; Marshall y Stolle, 2004).

La gestión de la Proximidad está orientada a desarrollar una acción socioeducativa contextualizada en el sí de la comunidad, articulando esfuerzos a partir de la inclusión de prácticas paralelas así como el trabajo altamente interdependiente con políticas locales (Agranoff y McGuire, 1999). De esta manera, se pretende que la RS responda a necesidades compartidas y objetivos comunitarios. La gestión de esta estrategia aspira a aumentar las sinergias y el compromiso socioeducativo con la búsqueda de alianzas a través de la comunidad (Gold et al., 2002). Otros autores señalan esta misma acción utilizando conceptos como activación -activating- (Agranoff y McGuire, 2001) o gestión política (Milward y Provan, 2006), centrada en las relaciones de la RS con su entorno. A su vez, esta estrategia pretende aumentar la participación de las personas de la comunidad en la RS mediante el desarrollo de canales de comunicación explícitos (Lipnack y Stamps, 1994) y de mecanismos concretos de afiliación y voluntarismo. Esta acción tiene como objetivo mejorar la confianza y la implicación de las personas en la comunidad con las problemáticas sociales (O'Leary, 2009). A su vez, la estrategia de Proximidad está relacionada con la variable de capital social centrada en el aumento de conexiones comunitarias, entendiendo que las comunidades que están más y mejor conectadas están más capacitadas para afrontar futuros retos (Burt, 2001).

Finalmente, la gestión de la Proyección incorpora mecanismos de planificación estratégica y evaluación con el objetivo de mejorar la calidad de la acción, así como su sostenibilidad y eficiencia. Esta estrategia dota a la acción de un componente preventivo a medio-largo plazo que capacita la RS evitando el desarrollo de una acción reactiva. Lipnack y Stamps (1994) apuntan que para garantizar la mejora continua del proyecto en la "Era de la Información y la Comunicación" es necesario el desarrollo de procesos de formación a los miembros, evaluaciones formales y el apoyo de las nuevas tecnologías. Shirley (2009) incorpora la importancia en la organización de comités o equipos de investigación, desarrollando acciones de investigación en diferentes momentos como por ejemplo analizar necesidades o acotar un problema. A su vez, esta estrategia engloba la promoción de espacios para debatir y proponer nuevas ideas y proyectos donde se busca el dinamismo y la innovación. La Proyección concreta dos variables específicas de capital social: la generación de conocimiento y la innovación. El intercambio de conocimientos entre agentes diversos y la generación de nuevos conocimientos compartidos aportan un valor tácito a los miembros de la red, mejorando las capacidades individuales y colectivas para solucionar problemas. Por otro lado, la inclusión de procesos de innovación, como procesos proactivos e intencionados para generar, implementar y diseminar nuevas y creativas ideas, tiene como objetivo promover un cambio 
cualitativo en un contexto particular (Sorensen y Torfing, 2011). La innovación colaborativa constituye un importante outcome en las RS, y se concreta articulando además de los recursos físicos y operativos de los miembros, la experticia y el talento de cada protagonista activo para formular continuamente nuevos proyectos y nuevas formas de organización.

\section{CONClusiones}

La gobernanza a nivel social y educativo vive actualmente un periodo de "crisis" y búsqueda de nuevas maneras sostenibles y eficaces de organizar los recursos y mejorar los resultados socioeducativos. El contexto socioeconómico actual, las limitaciones mostradas por programas socioeducativos tradicionales, así como la emergencia de nuevas formas de colaboración que pretenden dar respuesta a estas limitaciones a través de crear valor agregado, sitúan a las Rs como un reto apetecible para la gobernanza social y educativa a nivel comunitario. El presente trabajo hace un intenso recorrido sobre investigación nacional e internacional en gestión de RS para presentar el Modelo de Gestión Transversal de RS como estrategia de gobernanza socioeducativa en la comunidad. El modelo señala la necesidad de gestionar activamente tres dimensiones definidas - estructura, acción y cultura- para incrementar la efectividad de la acción. Seguidamente señala dos outcomes de proceso requeridos para incrementar su efectividad en el logro de objetivos: en primer lugar, el desarrollo de una cultura profesional colaborativa y, en segundo lugar, el incremento del capital social en la comunidad. Finalmente, el modelo concreta y profundiza sobre las estrategias necesarias para conseguir dichos outcomes y mejorar así los resultados socioeducativos planteados.

En este sentido, el trabajo sitúa la gestión activa para el desarrollo de una cultura colaborativa y del fortalecimiento comunitario, además de outcomes por sí solos, como prioridades para los gestores de la red en tanto que condiciones para el éxito. Entendemos que las claves actuales de la gobernanza socioeducativa no se basan simplemente en la implementación de nuevos proyectos en la comunidad, sino que integran el liderazgo del empoderamiento de las partes implicadas, mejorando las condiciones para la articulación de recursos y creando los mecanismos adecuados para desarrollar el compromiso y la participación de la comunidad en las metas socioeducativas. En una sociedad desconfiada (Fukuyama, 2001), donde además de un cierto desconocimiento entre los usuarios y los diferentes agentes socioeducativos de una misma comunidad se suma el desconocimiento entre los mismos servicios (servicios sociales, psicoeducativos, sanitarios o entre las propias escuelas), es relevante la necesidad de liderar propuestas que incrementen la confianza y el capital social en la comunidad para mejorar los resultados globales.

A su vez, el artículo presenta unas pautas significativas para la gestión de RS que, además de orientar su práctica, suponen un marco valioso para su propia evaluación. El Modelo de Gestión Transversal de RS acota y define seis estrategias y 
justifica la necesidad de centrar parte de los recursos de estas redes para mejorar su gestión. Este hecho contrasta con la realidad de muchas Rs de nuestro territorio, donde profesionales llevan a cabo las tareas de gestión de forma voluntaria e intuitiva. Tal y como hemos expuesto, la especificidad de la gestión de RS requiere profesionales capaces de asumir el complejo reto de gobernar una Rs. En futuros trabajos planeamos abordar la concreción de un marco de indicadores para evaluar el modelo y el impacto del mismo en la comunidad.

Finalmente, queremos destacar la importancia de promover el cambio hacia una cultura profesional basada en la colaboración, incluyendo las estrategias necesarias para sacar el máximo rendimiento a la misma. Éste es un aspecto clave, ya que la apertura de fronteras organizacionales y la interacción entre miembros diversos debe ir acompañada tanto de mecanismos para aprovechar y articular sus múltiples recursos, como de mecanismos para llevar a la práctica las nuevas respuestas. La idea de colaboración público-privada como motor de innovación en el ámbito social es lo que Sorensen y Torfing (2011) conocen como Innovación colaborativa. Entendemos que el cambio de la cultura profesional que proponen las Rs debe ir más allá de la mejora continua -evaluación y formación-, con el objetivo de llevar a la práctica nuevos proyectos que recojan la interdisciplinariedad y representatividad de la RS. De este modo, la gobernanza socioeducativa debe promover una cultura profesional basada en la innovación colaborativa para aumentar el capital social y responder a los nuevos retos de forma más transversal y dinámica. La innovación colaborativa como valor de las Rs es también un concepto a desarrollar en futuros trabajos desde una perspectiva de gestión.

\section{REFERENCIAS BIBLIOGRÁFICAS}

Agranoff, R. y McGuire, M. (1999) Expanding Intergovernmental Management's Hidden Dimensions. The American Review of Public Administration, 29 (4), 352-369.

- (2001) Big questions in public network management research. Journal of Public Administration Research and Theory, 11, 295-326.

- (2003) Collaborative Public Management; new strategies for local governments. Washington DC, Georgetown University Press.

Blasco, J. y CASADO, D. (2011) Avaluació dels Plans Educatius d'entorn. Ivàlua: Institut Català de Polítiques Públiques. Consultado el 15 de diciembre de 2011. http://www. ivalua.cat/documents/1/17_05_2011_10_04_09_informe_PEE_def.pdf.

Bourgault, J. y LaPierRe, R. (2000) Horizontality and Public Management. Canadian Centre for Management Development. Consultado el 15 de diciembre de 2011. http://www. csps-efpc.gc.ca/pbp/pub/pdfs/P96_e.pdf.

Bryson, J.; Crosby, B. y Stone, M. (2006) The design and implementation of cross-sector collaboratives: propositions from the literature. Public Administration Review, 66 (1), $44-55$.

BurT, R. (2001) New Directions in Economic Sociology. New York, Russell Sage Foundation.

Carpenter, H.; Cummings, C.; Dyson, A.; Jones, L.; Laing, K.; Oseman, D. y TodD, L. (2010) Extended services evaluation: end of year one report. Research report. London, DfE. 
Consultado el 20 de diciembre de 2011. https://www.education.gov.uk/publications/ eOrderingDownload/DFE-RR016.pdf.

Cigler, B. (1999) Pre-conditions for the emergence of multicommunity collaborative organizations. Policy Studies Review, 16 (1), 87-102.

Dente, B.; BobBio, L. y SPADA, A. (2005) Government or Governance of Urban Innovation. DIPS, 162, 1-22.

Díaz-Gibson, J. y Civís, M. (2011) Redes socioeducativas promotoras de Capital Social en la comunidad: un marco teórico de referencia. Cultura y Educación. Fundación Infancia y Aprendizaje, 23 (3), 415-429.

DíAz-Gibson, J.; Civís, M. y Longás, J. (2013) Transversal management in Educative Governance Networks: The Challenge of Comprehensive Education. Artículo en revisión (no publicado).

Díaz-Gibson, J.; Civís, M.; Longás, J. y López, A. (2010) The Study of Educative Network organization in the City of Barcelona, Spain: The Nou Barris District. International Journal of Knowledge Society Research, 1 (2), 26-37.

Edelenbos, J. y Klijn, H. (2006) Managing Stakeholder Involvement in Decision Making: A Comparative Analysis of Six Interactive Processes in the Netherlands. Journal of Public Administration Research and Theory, 16 (3), 417-446.

Eggers, B. y Singh, S. (2009) The Public Innovators Playbook: Nurturing Bold Ideas in Government. Washington DC, Ash Institute.

FucuYama, F. (2001) Social Capital, civil society and development. Third World Quarterly, 22 (1), 7-20.

Gold, E.; Simon, E. y Brown, C. (2002) Strong Neighborhoods, Strong Schools: Successful Community Organizing for School Reform. Chicago, Cross City Campaign for Urban School Reform.

GRAY, B. (2000) Assessing inter-organizational collaboration: Multiple conceptions and multiple methods, en FaulKner, D. y De Rond, M. (eds.) Perspectives on collaboration. New York, Oxford University Press, 243-260.

Hajer, M. y WagenaAr, H. (2003) Deliberative Policy Analysis: Understanding Governance in the Network Society. Cambridge, Cambridge University Press.

HJERn, B. (1992) Illegitimate Democracy: a Case for Multiorganizational Policy Analysis. Policy Currents, 2, 1-5.

KAmEnsky, A.; Burlin, T. y ABramson, M. (2004) Collaboration: Using Networks and Partnerships. Oxford, Rowman \& Littlefield Publishers, Inc.

KiCKeRT, W.; Klijn, E. y Koppenjan, J. (1997) Managing Complex Networks: Strategies for the Public Sector. London, Sage.

Klijn, E.; Edelenbos, J. y Steijn, B. (2010) Trust in Governance Networks: Its Impacts on Outcomes. Administration and Society, 42 (2), 193-221.

KreBs, V. y Holley, J. (2004) Building sustainable communities through social network development. The Nonprofit Quarterly, 11, 46-53.

LiPNaCK, J. y STAmPS, J. (1994) The Age of the Network. New York, Wiley.

López, M. (2008) Redes de apoyo para promover la inclusión educativa: Una revisión de algunos equipos y recursos. REICE: Revista Electrónica Iberoamericana sobre Calidad, Eficacia y Cambio en Educación, 6 (2), 200-211.

Mandell, M. y SteElman, T. (2003) Understanding what can be accomplished through interorganizational innovations: The importance of typologies, context and management. Public Management Review, 5 (2), 197-224. 
Marshall, M. y Stolle, D. (2004) Race and the City. Neighborhood Context and the Development of Generalized Trust. Political Behaviour, 26 (2), 125-154.

MediratTA, K.; SHAH, S. y McAlister, S. (2009) Community Organizing for Stronger schools: Strategies and Successes. Cambridge, Harvard Education Press.

Milward, B. y Provan, K. (2006) A Manager's Guide to Choosing and Using Collaborative Networks. IBM Endowment for the Business of Government. Consultado el 20 de diciembre de 2011. http://www.businessofgovernment.org/pdfs/ProvanReport.pdf.

O'Leary, R. y Bingham, L. (2009) The collaborative public manager: New ideas for the twenty first century. Whashington DC, Georgetown University Press.

Provan, K.; Fish, A. y SYDOw, J. (2007) Interorganizational Networks at the Network Level: A Review of the Empirical Literature on Whole Networks. Journal of Management, 33 (3), 479-516.

Provan, K. y Kenis, P. (2008) Modes of Network Governance: Structure, Management, and Effectiveness. Journal of Public Administration Research \& Theory, 18 (2), 229-252.

PROVAN, K. y MilwaRD, B. (1995) A Preliminary Theory of Network Effectiveness: A Comparative Study of Four Mental Health Systems. Administrative Science Quarterly, 40 (1), 1-33.

Putnam, R. (2000) Bowling alone, the collapse and revival of American community. New York, Simon \& Shuster.

RaINeY, H. (2009) Understanding and Managing Public Organizations. San Francisco, JosseyBass.

RenÉE, M. y MCAlister, S. (2011) The Strengths and Challenges of Community Organizing as an Education Reform Strategy: What the Research Says. Annenberg Institute for School Reform at Brown University. Consultado el 20 de diciembre de 2011. http://www. annenberginstitute.org/Products/coe.php.

SAZ-CARranza, A. y Ospina, S. (2010) The Behavioral Dimension of Governing Interorganizational Goal-Directed Networks: Managing the Unity-Diversity Tension. Journal of Public Administration Research and Theory, 21 (2), 327-365.

SCEARCE, D.; KASPER, G. y McLEOD, H. (2010) Working wikily 2.0. Standford Social Innovation Review, 8, 31-37.

SHIRLEY, D. (2009) Community Organizing and Educational Change: A Reconnaissance. Journal of Educational Change, 10, 229-237.

SCHÖn, D. (1998) El profesional reflexivo. Cómo piensan los profesionales cuando actúan. Barcelona, Paidós.

Skelcher, C. y Torfing, J. (2010) Improving democratic governance through institutional design: Civic participation and democratic ownership in Europe. Regulation and Governance, 4 (1), 71-91.

SORENSEN, E. y TORFING, J. (2007) Theories of democratic network governance. Cheltenham, Edward Elgar.

- (2009) Making Governance Networks effective and democratic through metagovernance. Public Administration, 87 (2), 234-258.

- (2011) Enhancing Collaborative Innovation in the Public Sector: An Analytical Framework. Administration \& Society, 43 (8), 842-868.

Stolle, D. y Rochon, D. (1998) Are all associations alike? Member diversity, associational type and the creation of social capital. American Behavioral Scientist, 42 (1), 47-65.

Tough, P. (2008) Whatever it takes: Geoffrey Canada's quest to change Harlem and America. New York, Houghton Mifflin Harcourt.

Ubieto, J. (2009) El Trabajo en Red. Usos posibles en Eduación, Salud Mental y Atención social. Barcelona, Gedisa. 